\title{
BMJ Open Interactive voice response interventions targeting behaviour change: a systematic literature review with meta-analysis and meta-regression
}

\author{
Stergiani Tsoli, ${ }^{1}$ Stephen Sutton, ${ }^{2}$ Aikaterini Kassavou ${ }^{2}$
}

To cite: Tsoli S, Sutton S, Kassavou A. Interactive voice response interventions targeting behaviour change: a systematic literature review with meta-analysis and meta-regression. BMJ Open 2018;8:e018974. doi:10.1136/ bmjopen-2017-018974

- Prepublication history and additional material for this paper are available online. To view these files, please visit the journal online (http://dx.doi. org/10.1136/bmjopen-2017018974).

Received 3 August 2017 Revised 14 November 2017 Accepted 16 November 2017

Check for updates

${ }^{1}$ Department of Public Health and Primary Care, School of Clinical Medicine, University of Cambridge, Cambridge, UK ${ }^{2}$ Primary Care Unit, Behavioural Science Group, Institute of Public Health, School of Clinical Medicine, University of Cambridge, Cambridge, UK

Correspondence to

Stergiani Tsoli;

s.tsoli@cantab.net

\section{ABSTRACT}

Objective A number of promising automated behaviour change interventions have been developed using advanced phone technology. This paper reviewed the effectiveness of interactive voice response (IVR)-based interventions designed to promote changes in specific health behaviours.

Methods A systematic literature review of papers published between January 1990 and September 2017 in MEDLINE, CINAHL, Embase, PsycINFO, SCOPUS and the Cochrane Central Register of Controlled Trials (CENTRAL) was conducted. From the total of 2546 papers identified, 15 randomised control trials (RCTs) met the eligibility criteria and were included in a random effects metaanalysis. Meta-regression analysis was used to explore whether behaviour change techniques (BCTs) that were used in the interventions were associated with intervention effectiveness.

Results Meta-analysis of 15 RCTs showed that IVRbased interventions had small but significant effects on promoting medication adherence $(\mathrm{OR}=1.527,95 \% \mathrm{Cl}$ 1.207 to $1.932, k=9, p=0.000$ ) and physical activity (Hedges' $\mathrm{g}=0.254,95 \% \mathrm{Cl} 0.068$ to $0.439, \mathrm{k}=3, \mathrm{p}=0.007$ ). No effects were found for alcohol (Hedges' $g=-0.077$, $95 \% \mathrm{Cl}-0.162$ to $0.007, \mathrm{k}=4, \mathrm{p}=0.073$ ) or diet (Hedges' $\mathrm{g}=0.130,95 \% \mathrm{Cl}-0.088$ to $0.347, \mathrm{k}=2, \mathrm{p}=0.242$ ). In the medication adherence studies, multivariable meta-regression including six BCTs explained $100 \%$ of the observed variance in effect size, but only the BCT 'information about health consequences' was significantly associated with effect size ( $\beta=0.690, \mathrm{SE}=0.199,95 \% \mathrm{Cl}$ 0.29 to $1.08, p=0.000$ )

Conclusion IVR-based interventions appear promising in changing specific health behaviours, such as medication adherence and physical activity. However, more studies are needed to elucidate further the combination of active components of IVR interventions that make them effective and test their feasibility and effectiveness using robust designs and objective outcome measures.

\section{INTRODUCTION}

Health-related behaviours have enormous impact on some of the leading causes of mortality and morbidity. ${ }^{12}$ Evidence show that behaviours such as non-adherence to medications, lack of physical activity, smoking,
Strengths and limitations of this study

This is the first review to examine the effectiveness of interactive voice response (IVR) interventions designed to promote changes in specific health behaviours and to investigate the active ingredients associated with intervention effectiveness.

- The findings of this review can inform the development of future IVR interventions targeting specific health behaviours.

- Given the small number and low quality of the studies included in the meta-analysis, the results should be treated with caution.

high consumption of alcohol and poor diet can cause a number of health conditions. ${ }^{3}$ National recommendations suggest that health services aiming to prevent and manage long-term health conditions should include initiatives to change health behaviours. Health-related behaviours are highly influenced by people's environmental context and sociocultural circumstances. However, these factors may be difficult to alter, at least in the short term. Healthcare initiatives to change behaviours in individuals or groups might be easier to implement and to evaluate. ${ }^{4}$

The rapidly evolving field on the use of emerging technologies in health interventions and especially in interventions targeting behaviour change appears promising ${ }^{5}$ because of their potential to reach a large number of people and in different contexts. The application of such technology in primary care can complement the physician's efforts to engage participants in an intervention, especially in a challenging setting, such as healthcare in rural and remote areas. ${ }^{6}$ In some cases, such interventions can diminish the barriers reported in interventions delivered face to face such as literacy or the disclosure of personal and sensitive information. 
Interactive voice response (IVR) is a novel phonebased platform that can be used to deliver health behaviour interventions. The definitions and descriptions of IVR systems vary significantly given the plethora of the systems developed for various purposes. A simplified description of an IVR system is that it is a telephone-based technology that uses touch-tone phones to enable the user to interact with the system using the telephone keypad as the interface where the human speaker is replaced by high-quality recorded interactive script. ${ }^{7}$ In more recent studies, software for voice recognition has been applied that might further enhance the acceptability ${ }^{8}$ and the effectiveness of the IVR. ${ }^{9}{ }^{10}$ Participants can either interact in real time with the IVR system or receive and make IVR calls.

This technology offers several advantages, including convenience, simplicity and confidentiality. ${ }^{11}$ One of the major benefits is the significant cost savings compared to usual care. ${ }^{12}$ A number of systematic literature reviews have documented the incremental effects resulting from the use of these technologies in healthcare settings. ${ }^{101113}$ A recent meta-analysis on telecommunication interventions (including IVR) found such technologies to be effective in promoting adherence to cardiometabolic medications. ${ }^{14}$ A recent Cochrane review on the effectiveness of automated telephone communication systems for preventing disease and managing long-term conditions concluded that these interventions can change patients' behaviours and improve clinical outcomes. ${ }^{15}$

Although informative, previous reviews described interventions delivered using different telephone communication systems (eg, voice messages and short text messages) and multiple delivery modes (eg, voice messages and access to a healthcare advisor) and included heterogeneous outcome measures. These are some of the elements that made these interventions complex and the investigation of their active and effective content challenging. To address this gap, the present review aimed to conduct a meta-analysis of the effectiveness of IVR interventions on different health behaviours and to identify the components of these interventions that are associated with their effectiveness. The findings could inform the development of future IVR interventions.

The taxonomy of behaviour change techniques (BCTs) has been widely used to classify the active intervention content that impacts on intervention effectiveness. ${ }^{16}$ The use of specific techniques, either singly or in combination, have been associated with intervention effectiveness, even in people of lower socioeconomic backgrounds. ${ }^{17}$ However, little is currently known about which BCTs may be associated with the effectiveness of IVR-based interventions. Thus, this review aimed to address this question.

To our knowledge, this is the first attempt to summarise the most rigorous evidence on the effectiveness of IVR-based interventions to change health behaviours and to examine whether and what BCTs are associated with intervention effectiveness.

\section{METHODS}

\section{Search strategy and inclusion and exclusion criteria}

Six electronic databases (MEDLINE, CINAHL, Embase, PsycINFO, SCOPUS, Cochrane Central Register of Controlled Trials (CENTRAL)) were searched for studies published in peer-reviewed journals from January 1990 to September 2017. A detailed search strategy was constructed and run with the support of a medical librarian, including free text and Medical Subject Headings terms according to availability. Grey literature was not searched (see online supplementary file 1, for detailed search strategy for each database).

Adults ( $\geq 18$ years of age) were eligible for inclusion. Given the range of descriptions of the IVR delivery mode identified through the screening process, we decided to adopt a broad definition of IVR interventions and include those that consisted of an automated, computer-based system, delivering the intervention content through a telephone call that facilitated interactive or non-interactive voice messages. We included studies that used a randomised controlled or cluster randomised controlled design to test the effectiveness of such an intervention in any setting and that were published in English (online supplementary file 2).

We excluded studies when the main content of the intervention was delivered using other delivery modes, such as text messages, counselling sessions with, or follow-up calls from a healthcare provider (eg, please see references 18 19), and the impact of the IVR content on the intervention effectiveness could not be calculated. We also excluded studies that used IVR only as a way to collect outcome measures. Similarly, we excluded studies that compared the IVR intervention with another active type of intervention (eg, tailored booklet).

The aim of this review was to identify evidence for the effectiveness of IVR-based interventions and inform the development of an IVR-based intervention as an adjunct to primary care consultations. Thus, we included studies only when they provided evidence about the effectiveness of the IVR intervention itself compared to usual care or a non-active intervention group.

\section{Study selection}

Two reviewers independently screened studies by title and abstract according to the eligibility criteria. Agreement between reviewers at this stage was good (kappa=0.6). A third reviewer was available to discuss any disagreements. To identify any additional studies that fulfilled the inclusion criteria, two reviewers independently screened the references of all the eligible papers. The three reviewers independently screened studies for eligibility at full text. Authors of the eligible studies and those of the included studies were contacted via email to request missing information. Any disagreements were discussed until consensus was reached among the three reviewers. The review protocol was not registered. 


\section{Data extraction and intervention coding}

An extraction form was developed to extract the following information: the author, year, sample size, participant characteristics, description of the intervention and comparator groups, duration and content of the IVR intervention, outcome measures and outcome data.

For the alcohol outcome, we extracted the frequency and quantity of standard units per extended period of time (eg, per week). Alcohol consumption was defined as drinking regularly more than 14 units per week. Heavy alcohol consumption or binge drinking was defined as drinking 4-5 standard units per occasion. ${ }^{20}$ For the diet outcome, we extracted data measuring dietary patterns, such as frequency of consumption of recommended levels of food (eg, fruits and vegetables $\geq 5$ servings) and overall quantity of consumed food (eg, servings of fruit and vegetables, per cent of total calories and grams of fibre). Unhealthy diet was defined as consuming (a) less than five fruits and vegetables per day and/or (b) more than $30 \mathrm{~g}$ (for men) or $20 \mathrm{~g}$ (for women) of saturated fat per day, $5 \mathrm{~g}$ added sugar per $100 \mathrm{~g}$ and $6 \mathrm{~g}$ sodium per day. ${ }^{21}$ For the physical activity outcome, we extracted the minutes of moderate or greater intensity of physical activity. Physical inactivity was defined as taking less than (a) $150 \mathrm{~min}$ of moderate activity in bouts of $10 \mathrm{~min}$ and over on at least 5 days a week or (b) 75 min of vigorous activity spread across the week or a combination of both moderate and vigorous physical activity. ${ }^{22}$ For the medication taking outcome, we extracted the percentage of tablets taken as prescribed and days of medication dispensed to patients. Medication adherence was defined as (a) taking at least $80 \%$ of the prescribed tablets and/or (b) having medications dispensed for at least $95 \%$ days of a prescribed period. ${ }^{23}$

We extracted the method to collect each outcome, such as self-reported scales (for alcohol, diet, physical activity and medication adherence), accelerometers (for physical activity) and pill count or electronic pharmacy records (for medication adherence). Two reviewers independently assessed risk of bias using an adapted version of the Cochrane Collaboration tool for assessing risk of bias $^{24}$ (online supplementary file 3).

We coded the BCTs delivered by the IVR delivery mode using the Taxonomy V.1. ${ }^{16}$ We coded the BCTs delivered using other delivery modes (eg, letter), only if the content of this additional mode informed or was informed by the content of the IVR. For example, in Helzer et $a l^{6}$ study, ${ }^{6}$ the BCT 'feedback on behaviour' was coded, although delivered through a bar graph showing the number of drinks, because the graph was informed by participants' reports to the IVR system. We coded the intervention content as 'personalised' when it used the participant's name, and as 'tailored' when it delivered different content to different participants. We coded one additional technique: 'report whether or not the behaviour was performed', when participants were asked to report (non) performance of the behaviour to the IVR system. When there was more than one intervention condition and the intervention content was different between these conditions (ie, one intervention condition included different BCTs from the other intervention condition), we included the condition that used the greater number of IVR BCTs (eg, Andersson $2015^{25}$ ). The three reviewers independently coded the BCTs. Any disagreement in BCT coding was discussed until agreement was reached between reviewers.

\section{Data synthesis and meta-analyses}

To calculate the effect size, we used the unadjusted values at the end of the intervention for the intervention and comparator groups. This was decided based on the assumption that measurements at the baseline could have a reactive impact on intervention effectiveness, ${ }^{26}{ }^{27}$ thus to minimise this effect, only follow-up data were included in the analysis. For continuous data, we used the means, standard deviations (SD) and sample sizes. For dichotomous data, we used the number of participants who performed the behaviour and the total sample size. Medication adherence effect sizes are reported as odd ratio (OR) and 95\% confidence intervals (CIs). Alcohol, diet and physical activity effect sizes are reported as Hedges' $\mathrm{g}$ and $95 \%$ CI.

When more than one intervention group was included in a study and the content of these intervention groups was similar, ${ }^{6}$ the outcome data from the intervention groups were pooled and compared with the comparator group. This was done for Helzer et at study, where the two intervention groups were similar in terms of content, with the only difference being the financial incentives to increase compliance to one of the intervention BCTs.

A random effects meta-analysis was conducted for each behaviour (alcohol consumption, diet, physical activity and medication adherence) to estimate the overall effect size. A random effects model was chosen as it allows the true effect size to vary across studies.

\section{Assessment of heterogeneity}

The degree of heterogeneity was assessed using the $\mathrm{I}^{2}$ statistic. ${ }^{28}$ A value of $\mathrm{I}^{2}$ of $25 \%$ was considered as indicating low heterogeneity, $50 \%$ moderate and $75 \%$ high heterogeneity. ${ }^{24}$

\section{Analysis by study size}

A subgroup analysis tested whether the study size impacted on overall effect size by comparing the effect between smaller and larger studies.

\section{Publication bias}

The presence of possible publication bias was assessed by funnel plot, plotting precision (study-specific standard error of the estimate) against the effect size (Hedges' g). Asymmetry in the overall pattern was assessed using Egger's test by regressing the effect size on its standard error (SE). ${ }^{29}$

\section{Meta-regression analyses}

In addition, the following meta-regression analyses were conducted: 
A. A series of univariable meta-regression analyses, testing whether the intervention effect differs if a particular BCT was present or absent.

B. A multivariable meta-regression analysis, examining which BCTs were associated with the intervention effect.

The Comprehensive Meta-analysis software was used to perform all analyses. ${ }^{30}$ Statistical significance was set at $\mathrm{p}<0.05$.

\section{RESULTS}

\section{Search results}

The electronic searches yielded in total 5541 publications. After duplicate records were removed, title and abstract screening was performed on 2546 studies. At this stage, papers were excluded if they failed to meet the inclusion criteria of RCT study design, usual care or no-active comparator group, content and behavioural outcome, and use of an IVR delivery mode. In total, 207 publications were considered relevant for full-text inspection, of which $15^{62531-43}$ were included in the meta-analyses (figure 1). The rest of the studies ${ }^{44-50}$ did not provide comparable data to be included in the meta-analysis and meta-regression. Included studies targeted changes in medication adherence, physical activity, diet and alcohol consumption. No study on smoking cessation provided sufficient data to be eligible for analysis.

\section{Description of the included studies}

The majority of the studies targeted changes in a single behaviour; eight targeted medication adherence, ${ }^{31-38}$ four targeted alcohol behaviour ${ }^{6253943}$ and one targeted physical activity. ${ }^{40}$ Two studies ${ }^{4142}$ targeted changes in more than one behaviour: one targeted simultaneously diet and physical activity ${ }^{41}$ and the other diet, physical

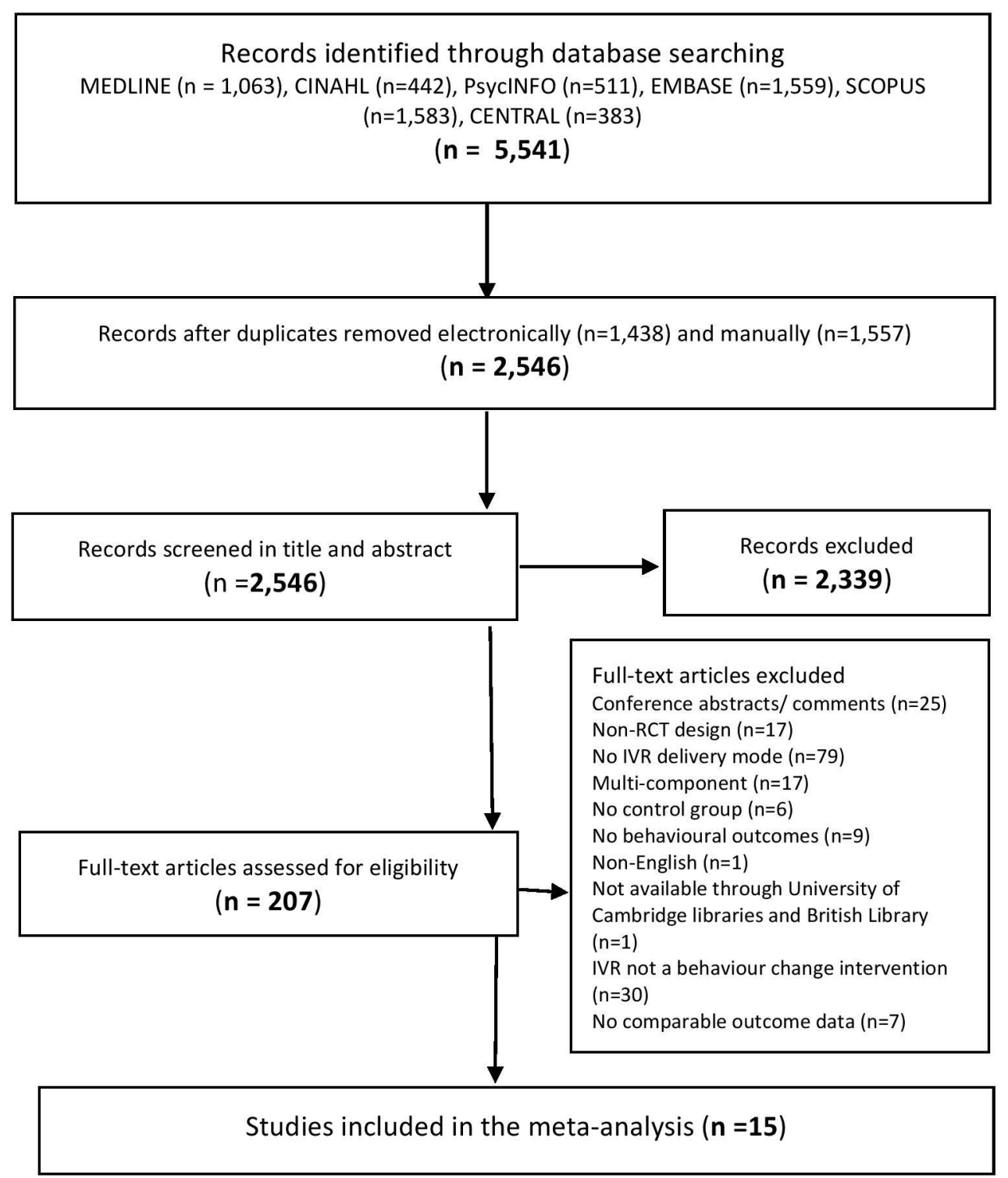

Figure 1 PRISMA flow chart of screening process. IVR, interactive voice response; PRISMA, Preferred Reporting Items for Systematic Reviews and Meta-Analyses; RCT, randomised controlled trials. 
activity and medication adherence. ${ }^{42}$ In total, four investigations in alcohol consumption, two in dietary behaviour, three in physical activity and nine in medication adherence were identified.

All of the included studies were RCTs, as no eligible cluster RCTs were identified through the screening process. The majority of the studies were undertaken in the USA, ${ }^{6} 31-333637$ 39-43 two studies in Canada, ${ }^{35} 38$ one in Sweden ${ }^{25}$ and one study in India. ${ }^{34}$

The total number of randomised participants was 35257 (ranging from $77^{41}$ to $14502^{33}$ ), with a total of 27867 completing follow-up measurements. All included trials investigated the impact of the intervention in groups of adults (range from 17.5/18 to 98 years old) (table 1).

\section{Quality assessment of the included studies}

Methodological quality of the included studies widely varied. Despite the fact that all the included studies used an RCT design, assessment of the randomisation process and allocation concealment was challenging for the majority of the studies due to lack of information provided. Blinding of participants was impossible due to the nature of the intervention. Six studies ${ }^{31-33363741}$ used objective outcome measures for all (medication adherence $^{31-3336{ }^{37}}$ ) or some of the behavioural outcomes (physical activity only). ${ }^{41}$ Two studies ${ }^{40}$ used objective outcome measures (ie, accelerometer for physical activity) in a subsample of participants to validate self-report measures. On average, fewer than half of the criteria were met, resulting in a review with included studies having a relatively high risk of bias (table 2 ).

\section{Characteristics of the delivery mode: intervention group}

Several different kinds of IVR systems were used in the included studies, and different names were used to describe them. The system was described as an IVR system in nine studies ${ }^{25} 3133$ 35-38 4143 and as an Alcohol Therapeutic IVR in one study. ${ }^{39}$ Other names used were automated Telephone Linked Computer system, ${ }^{40}$ Telephone-Linked Care ${ }^{42}$ and automated telephone calls. ${ }^{32} 34$ The IVR delivery mode was quite variable in structure and interaction with the participant. For instance, the intervention described by Derose $e t a l^{32}$ had few interactive features. Call duration ranged from $40 \mathrm{sec}^{32}$ to $10 \mathrm{~min}$ maximum. ${ }^{41}$ However, only seven studies provided this kind of information (table 1).

\section{Description of the BCTs by behaviour: intervention condition Alcohol consumption}

In interventions targeting alcohol consumption, the most frequently coded BCTs were 'tailored' and 'report whether or not the behaviour was performed', all coded in four studies; 'feedback on behaviour' coded in three studies; 'avoidance/reducing exposure to cues for behaviour', 'behaviour substitution', 'credible source', 'discrepancy between current behaviour and goals', 'goal setting (behaviour)', 'personalised' and 'social support (unspecified)', each coded in two studies; 'distraction', 'framing/ reframing', 'graded task', 'information about health consequences', 'information about social and environmental consequences', 'instructions on how to perform the behaviour', 'problem solving', 'prompts/cues', pros and cons', 'reduce negative emotions', 'relapse prevention and coping planning', 'restructuring the social environment', 'review behavioural goals', 'social comparison', 'social reward', 'social support(practical)' and 'social support (emotional)', each coded in one study.

\section{Dietary behaviour}

In interventions targeting dietary behaviour, the following BCTs were coded in each study: 'tailored', 'social support (unspecified)', 'self-monitoring of outcome(s) of behaviour', 'prompts/cues', and 'action planning'.

\section{Physical activity}

In interventions targeting physical activity, the BCTs 'tailored' and 'self-monitoring of behaviour' were coded in two studies. Each of the BCTs 'prompts/cues', 'action planning', 'social support (unspecified)', 'problem solving' and 'reporting whether or not the behaviour was performed' were coded in one study.

\section{Medication adherence}

In interventions targeting medication adherence, the BCT 'social support (unspecified)' was coded in eight studies; 'prompts/cues' in five studies; 'tailored' and 'information about health consequences' each coded in four studies; 'personalised' and 'report whether or not the behaviour was performed', each coded in three studies; 'social reward' was coded in two studies; and 'feedback on behaviour' and 'problem solving' each coded in one study

\section{Characteristics of the delivery mode and content: comparator condition}

Most studies did not provide a detailed description of the comparator condition. The comparator condition was most commonly described as 'usual care' but without specifying the content and, in some cases, the delivery mode of this care. One study ${ }^{34}$ mentioned that the usual care consisted of three counselling sessions, laboratory and clinical tests. The comparator condition consisted of a health education class on non-physical activity topics in one study, ${ }^{40}$ a diabetes prevention class in another study ${ }^{41}$ and one IVR call with general advice on health and a guide via email in another study. ${ }^{36}{ }^{36}$ No BCTs delivered via IVR were coded in the comparator condition.

\section{Meta-analyses}

The meta-analysis of included studies demonstrated that IVR-based interventions had a small but statistically significant effect on increasing physical activity (Hedges' $\mathrm{g}=0.254,95 \%$ CI 0.068 to $0.439, \mathrm{k}=3, \mathrm{p}=0.007)$ and medication adherence (OR=1.527, 95\% CI 1.207 to 1.932, $\mathrm{k}=9, \mathrm{p}=0.000$ ). There was no statistically significant effect on behaviours related to diet (Hedges' $\mathrm{g}=0.130,95 \% \mathrm{CI}$ -0.088 to $0.347, \mathrm{k}=2, \mathrm{p}=0.242$ ) or alcohol consumption 


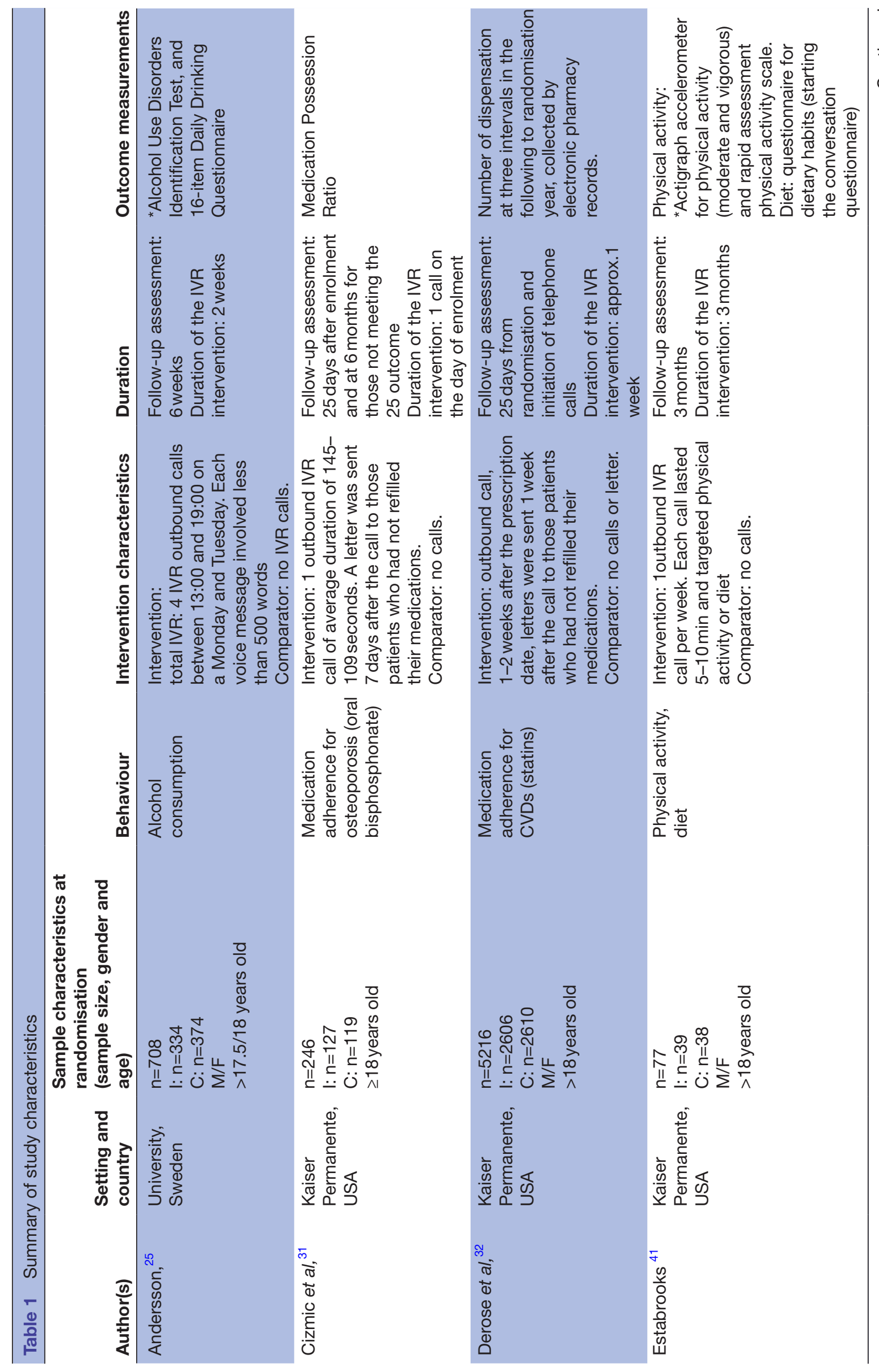

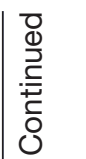




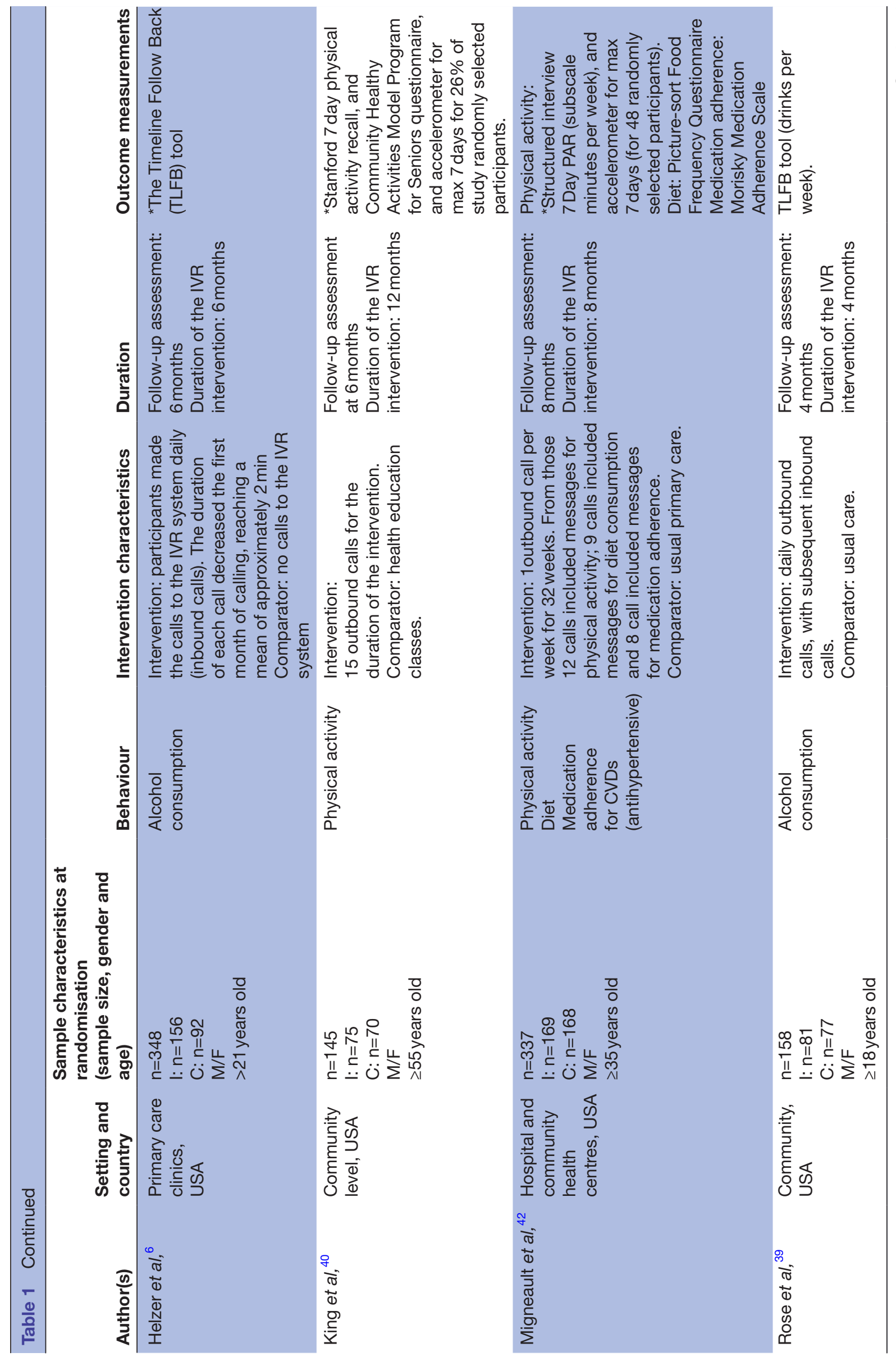

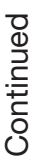




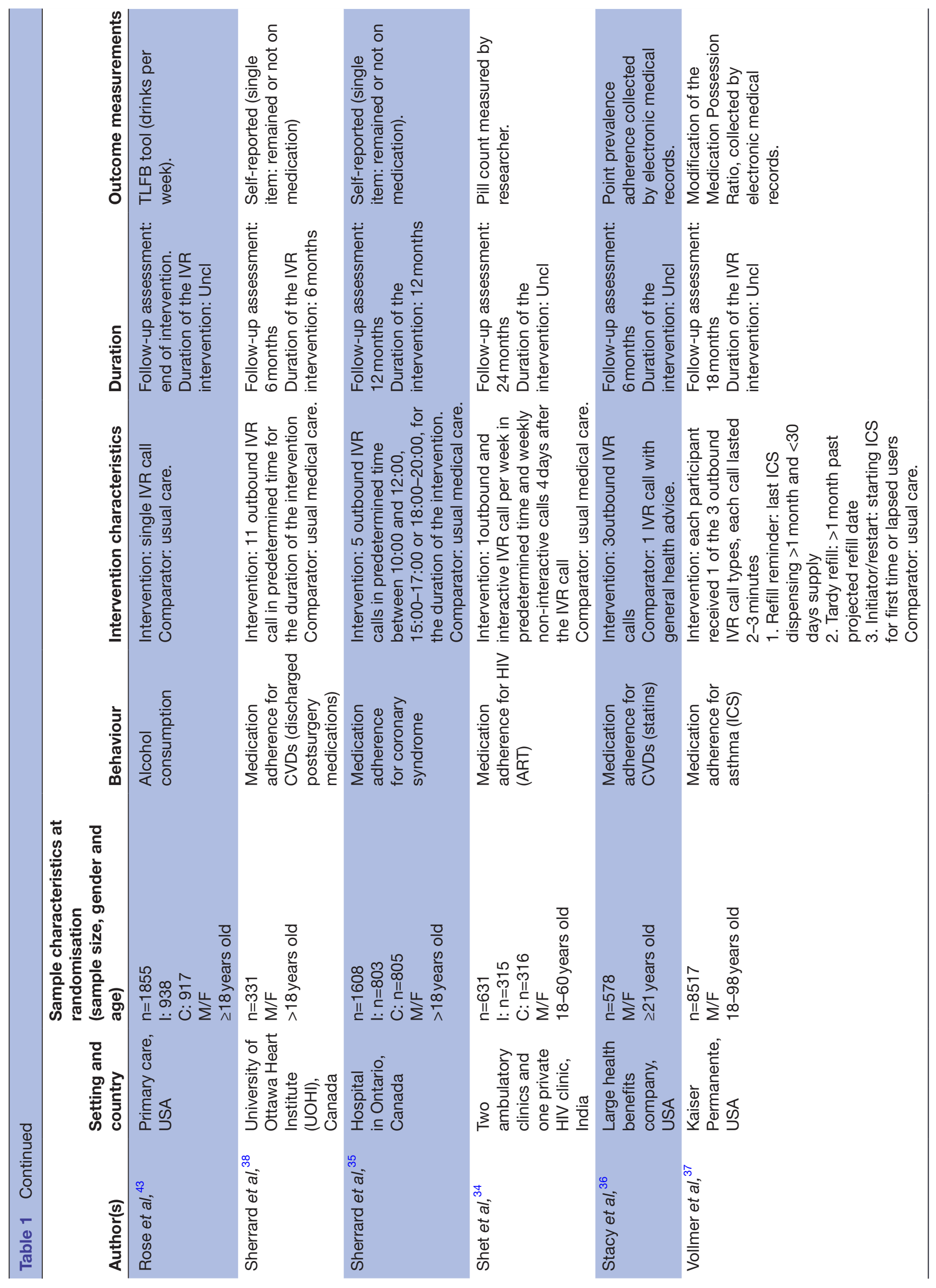

뭉

$\frac{\bar{c}}{\bar{c}}$ 
(Hedges' $\mathrm{g}=-0.077,95 \%$ CI -0.162 to $0.007, \mathrm{k}=4, \mathrm{p}=0.073$ ). No study reported statistically significant evidence in favour of the comparator condition compared to the IVR intervention (figure 2).

Studies targeting physical activity, alcohol and dietary behaviours were highly homogeneous (all had I ${ }^{2}=0.00 \%$ ), whereas studies targeting medication adherence were highly heterogeneous $\left(I^{2}=93.84 \%\right)$.

\section{Analysis by study size}

Subgroup analysis was conducted to examine any differences in the overall effect size due to differences in study sample size, and results showed no significant effects. Specifically, for diet interventions, the smaller size study had larger effect size (Hedges' g=0.285, 95\% CI -0.222 to $0.792, \mathrm{k}=1$ ) in comparison with the larger size study (Hedges' $\mathrm{g}=0.095,95 \%$ CI -0.146 to $0.335, \mathrm{k}=1$ ); however, that difference was not significantly different. For physical activity interventions, studies of smaller size had a larger effect size (Hedges' $\mathrm{g}=0.346,95 \%$ CI 0.055 to $0.636, \mathrm{k}=2$ ) in comparison to the large size study (Hedges' $\mathrm{g}=0.191$, $95 \% \mathrm{CI}-0.051$ to $0.432, \mathrm{k}=1$ ); however, that difference was not significantly different. For alcohol interventions, smaller size studies had a larger effect size (Hedges' $\mathrm{g}=-0.058,95 \%$ CI -0.150 to $0.034, \mathrm{k}=2$ ) in comparison to larger size studies (Hedges' $\mathrm{g}=-0.181,95 \%$ CI -0.395 to $0.032, \mathrm{k}=2$ ); however, that difference was not significantly different. For medication adherence interventions, studies of smaller size had a larger effect size $(\mathrm{OR}=1.86$, $95 \%$ CI 1.328 to $2.605, \mathrm{k}=4$ ) in comparison to studies of larger size ( $\mathrm{OR}=1.35,95 \%$ CI 1.009 to $1.820, \mathrm{k}=6)$; however, that difference was not significantly different.

\section{Publication bias}

Inspection of the funnel plot appears to suggest asymmetry in the plot, more pronounced in the larger studies (figure 3). However, Egger's test for asymmetry was not statistically significant $(\mathrm{p}=0.534)$. Nevertheless, the presence of funnel plot asymmetry could have resulted from other reasons apart from publication bias, ${ }^{51}$ such as participants' characteristics at baseline.

\section{Meta-regression analyses}

Due to the small number of studies on physical activity, alcohol and diet, it was not possible to explore what BCTs were associated with intervention effect sizes (online supplementary file 4 ).

Meta-regression analysis was performed only on the studies aiming to promote medication adherence. BCTs coded in at least three studies were included in the meta-regression analyses. Univariable meta-regression analysis of the medication adherence studies showed that the BCTs 'personalised' $\left(\tau^{2}=0.028, \mathrm{R}^{2}=0.73, \mathrm{p}=0.006\right.$ ), 'social support (unspecified)' $\left(\tau^{2}=0.101, \mathrm{R}^{2}=0.03, \mathrm{p}=0.043\right)$ and 'information about health consequences' $\left(\tau^{2}=0.003, R^{2}=0.97, p=0.000\right)$ were associated with larger effect sizes (online supplementary file 4 ).

Multivariable meta-regression analysis showed that the presence or absence of the six BCTs coded in medication 
Table 2 Risk of bias of included studies by behaviour

\begin{tabular}{|c|c|c|c|c|c|c|c|}
\hline Author, year & $\begin{array}{l}\text { Random } \\
\text { sequence } \\
\text { generation }\end{array}$ & $\begin{array}{l}\text { Allocation } \\
\text { concealment }\end{array}$ & $\begin{array}{l}\text { Blinding of participants, } \\
\text { personnel and outcome } \\
\text { assessors }\end{array}$ & $\begin{array}{l}\text { Incomplete } \\
\text { outcome } \\
\text { data }\end{array}$ & $\begin{array}{l}\text { Selective } \\
\text { reporting }\end{array}$ & $\begin{array}{l}\text { Objective } \\
\text { outcome } \\
\text { assessment }\end{array}$ & $\begin{array}{l}\text { Other } \\
\text { bias }\end{array}$ \\
\hline \multicolumn{8}{|l|}{ Alcohol consumption } \\
\hline Andersson, ${ }^{25} 2015$ & $?$ & $?$ & - & + & + & - & $?$ \\
\hline Helzer et al, ${ }^{6} 2008$ & $?$ & $?$ & - & + & - & - & $?$ \\
\hline Rose et al, ${ }^{39} 2015$ & $?$ & $?$ & - & $?$ & $?$ & - & + \\
\hline Rose et al, ${ }^{43} 2017$ & + & $?$ & + & $?$ & $?$ & - & + \\
\hline \multicolumn{8}{|l|}{ Medication adherence } \\
\hline Cizmic et al, ${ }^{31} 2015$ & + & $?$ & - & + & $?$ & + & + \\
\hline Derose et al, ${ }^{32} 2013$ & + & $?$ & - & + & $?$ & + & + \\
\hline Migneault et al, ${ }^{42} 2012$ & + & $?$ & - & $?$ & $?$ & - & + \\
\hline Sherrard et al, ${ }^{38} 2009$ & $?$ & + & - & + & - & - & + \\
\hline Sherrard et $a l,{ }^{35} 2015$ & + & $?$ & - & - & $?$ & - & + \\
\hline Shet et al, ${ }^{34} 2014$ & $?$ & + & - & + & $?$ & + & + \\
\hline Stacy et $a l,{ }^{36} 2009$ & $?$ & $?$ & - & + & $?$ & + & + \\
\hline Vollmer et al, ${ }^{37} 2011$ & $?$ & $?$ & - & + & $?$ & + & + \\
\hline Vollmer et al, ${ }^{33} 2014$ & + & $?$ & - & + & $?$ & + & + \\
\hline \multicolumn{8}{|l|}{ Diet } \\
\hline Estabrooks, ${ }^{41} 2008$ & $?$ & $?$ & - & + & $?$ & - & $?$ \\
\hline Migneault et al, ${ }^{42} 2012$ & + & $?$ & - & $?$ & $?$ & - & + \\
\hline \multicolumn{8}{|l|}{ Physical activity } \\
\hline Estabrooks, ${ }^{41} 2008$ & $?$ & $?$ & - & + & $?$ & + & $?$ \\
\hline King et al,$^{40} 2007$ & + & $?$ & - & + & $?$ & - & + \\
\hline Migneault et al, ${ }^{42} 2012$ & + & $?$ & - & $?$ & $?$ & - & + \\
\hline
\end{tabular}

Note: '+' indicates low risk of bias; '-' indicates high risk of bias; '?' indicates unclear risk of bias.

adherence studies explained $100 \%$ of the variance in intervention effect size. Moreover, the goodness of fit model was not statistically significant $\left(\tau^{2}=0.000, \tau=0.000\right.$, $\left.\mathrm{I}^{2}=0.00 \%, \mathrm{Q}=0.04, \mathrm{df}=2, \mathrm{p}=0.981\right)$, suggesting that the effect size does not vary across studies that used/did not use the same BCTs. The multivariable meta-regression model showed that the BCT 'information about health consequences' was positively associated with a larger intervention effect size, when the remaining five BCTs were controlled for $(\beta=0.690, \mathrm{SE}=0.199,95 \%$ CI 0.29 to $1.08, \mathrm{p}=0.000)$. None of the other five BCTs were significantly associated with intervention effectiveness (online supplementary file 4 ).

\section{DISCUSSION}

\section{Principal findings}

The results from this meta-analysis of 15 RCTs suggest that IVR-based interventions are efficacious in changing medication adherence and physical activity but not alcohol or dietary behaviours. No effect of study size on overall effect size was detected for any of the behaviours. Results from the univariable meta-regression in medication adherence interventions suggested that the BCTs 'personalised', 'social support (unspecified)' and 'information about health consequences' were associated with a larger effect. However, only the BCT 'information about health consequences' was significant in the multivariable meta-regression analysis. This finding suggests that IVR interventions that include information about the health consequences of (not) taking medications as prescribed, are personalised and provide social support could increase adherence to different types of medication. For the interventions targeting physical activity, the most frequently used BCTs were 'tailored' and 'self-monitoring of behaviour', although conclusions about their impact on intervention effectiveness could not be drawn. Similarly, conclusions about the use of the most frequently used BCTs and their impact on the non-efficacious alcohol and diet interventions could not be made.

\section{Strength and limitations}

Caution should be applied when interpreting the results of this review due to the low quality of the included studies. Despite this, studies of higher quality had positive effects on changing medication adherence and physical activity behaviours, and considering that these studies included objective outcome measurements or 

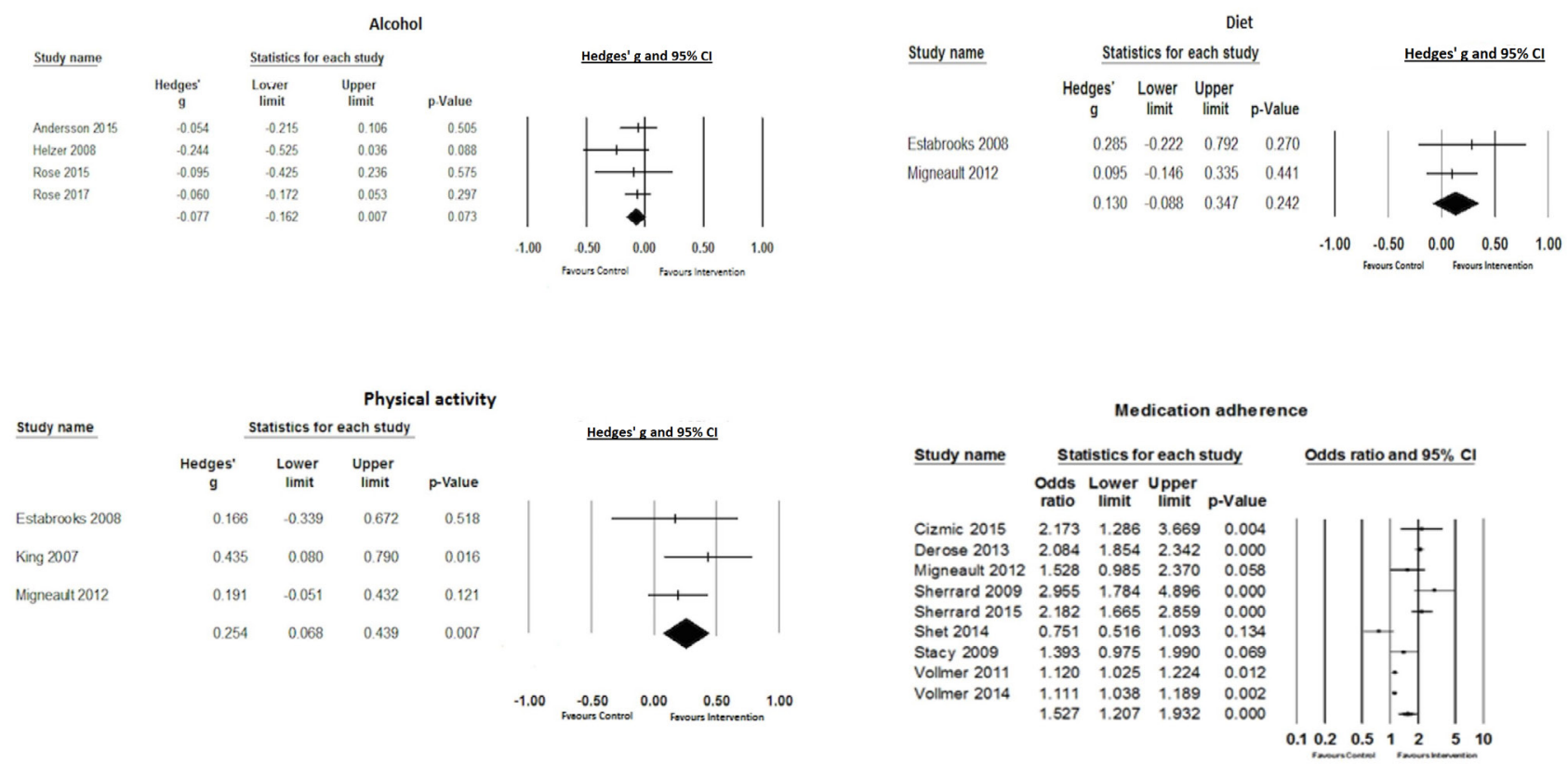

Figure 2 Forest plots of the Hedges' g (95\% Cl) for alcohol, diet and physical activity interventions and OR $(95 \% \mathrm{Cl})$ for the medication adherence interventions. Differences are presented between the intervention and a comparator condition.

their subjective measurements had been validated by objective measurements, such interventions could have a significant impact on public health if applied on a large scale.

However, seven eligible studies could not be included in the meta-analysis due to missing outcome data. Thus, the effects might have been slightly different from those estimated by the present analyses. Studies were excluded during full-text screening due to outcome measures not being comparable with the outcomes of interest. However, among the studies not included in the analysis, only one study ${ }^{47}$ did not confirm the benefits of IVR-based interventions. Grey literature was not searched; thus it is possible that some studies were missed. The meta-regression findings

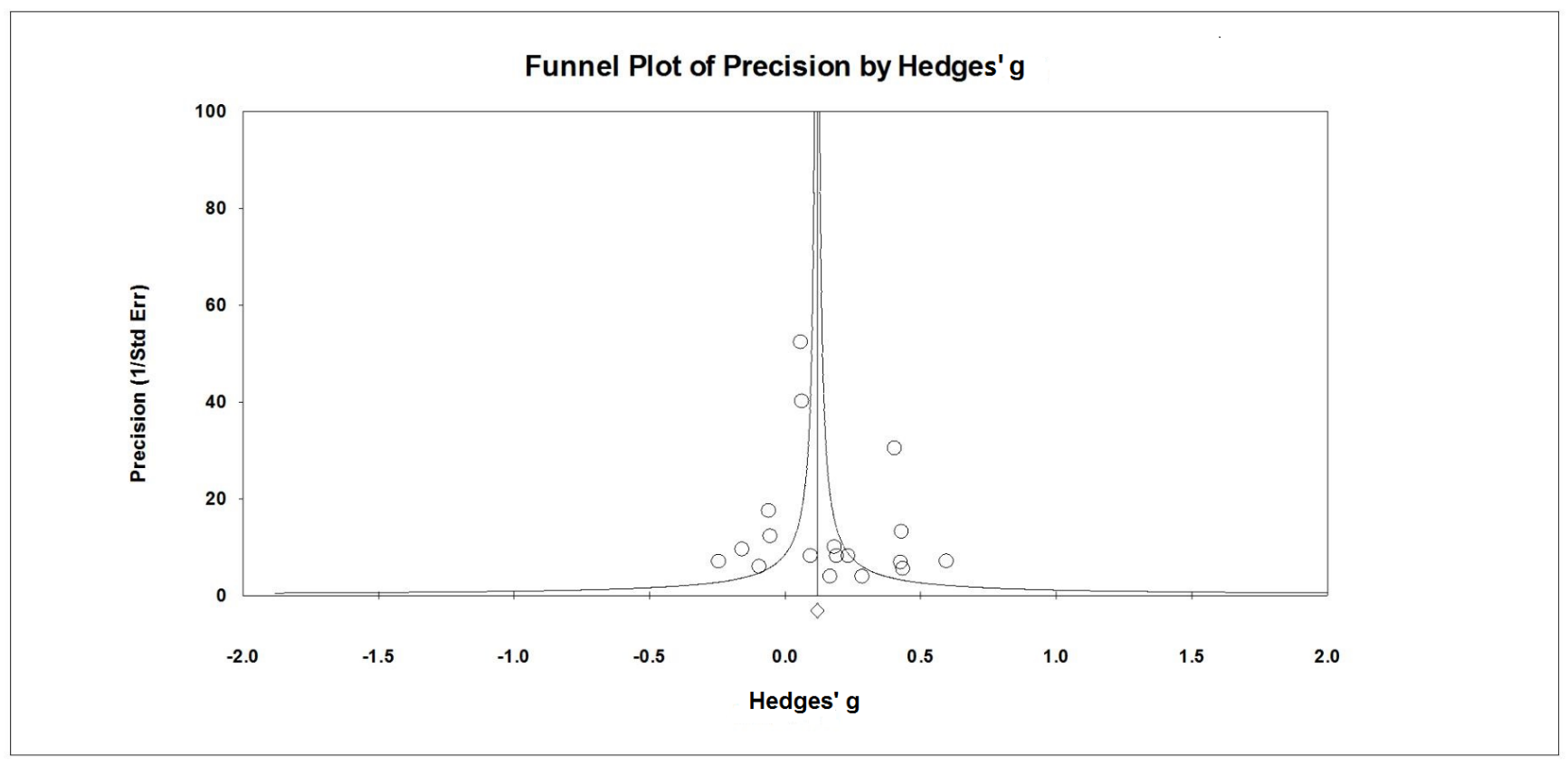

Figure 3 Funnel plot of precision against Hedges' g. 
also need to be interpreted with caution due to the small number of studies ${ }^{51}$ and the high correlation of the BCTs.

\section{How this review is different from previous studies}

To our knowledge, the current review is the first attempt to summarise the best available evidence on IVR-based interventions and investigate the intervention content that may be associated with their effectiveness; thus comparability with previous reviews is limited. The only comparable review is that by Posadzki $e t a l^{15}$ which suggested that IVR interventions are probably effective in producing small effects on alcohol, diet, physical activity and medication adherence. However, due to the small number and high heterogeneity of the included studies, Posadzki et al did not conduct meta-regression analysis to identify the active components of the interventions for specific behaviours.

To achieve this, the present review included interventions that were comparable in terms of intervention components, outcomes and outcome measures. The analysis of alcohol, diet and physical activity interventions showed that the effects were homogeneous $\left(\mathrm{I}^{2}=0.00 \%\right)$. Moreover, the meta-regression analysis on medication adherence interventions explained $100 \%$ of the variance in intervention effect. These findings could inform the development of future IVR interventions targeting changes in such health behaviours.

\section{Possible mechanisms and implications for future research}

The included IVR-delivered interventions varied in several elements of the delivery mode, which might have contributed to the estimated effects. For example, the degree of interaction with patients varied widely among the interventions. Some studies described a more interactive system that might have increased participants' engagement with the intervention (eg, $31{ }^{40}$ ). An interesting feature of some of the IVR interventions was the use of culturally sensitive voices (eg, 2542 ) or prerecorded messages of their therapist's voice to increase acceptability of the intervention. ${ }^{39}$ Furthermore, another study provided participants with the opportunity to choose the gender and language of the voice delivering the IVR intervention. ${ }^{34}$ These features of the delivery mode could have influenced engagement with the intervention and potentially impacted on its effectiveness.

Providing information about the health consequences appeared effective in promoting medication adherence. Such information usually includes the advantages of taking medications or the disadvantages of not taking them. This finding is in line with a recent review that investigated the BCTs that promote adherence to cardiometabolic medications. ${ }^{14}$ However, it further suggests that such a technique might be effective in promoting adherence to different types of cardiometabolic medication and to medications for different health conditions (eg, oral bisphosphonate for osteoporosis, antiretroviral treatment for HIV and inhaled corticosteroids for asthma). Social support (unspecified) was associated with larger intervention effect size. This finding is in line with previous research that suggests that types of perceived social support are significantly associated with medication adherence. ${ }^{52}$ It is also possible that IVR voice messages might increase the sense of social support, in comparison to other delivery modes, due their resemblance to 'natural' verbal communication ${ }^{40}$ or due to the availability of the IVR for patients to call in and ask for support.

For alcohol, dietary and physical activity interventions, some of the coded BCTs have previously been associated with intervention effectiveness: 'self-monitoring of behaviour' and 'action planning' for both dietary and physical activity interventions ${ }^{53}$ and 'feedback on behaviour' for alcohol interventions. ${ }^{54}$ However, due to the limited number of included studies, it was not possible to draw conclusions about the efficacy of these BCTs to promote behaviour changes.

Moreover, the primary studies did not provide a detailed description of the content and delivery mode for the comparator condition, which limits our ability to interpret the effect of the successful interventions. ${ }^{55} \mathrm{~A}$ number of other factors need to be further investigated when interpreting the findings of this review, such as participants' characteristics at baseline, the recruitment setting and the study design.

\section{CONCLUSION}

This review provides evidence about the effectiveness of IVR interventions to promote specific health behaviours and therefore promote public health. It also provides evidence on the BCTs that are associated with the effectiveness of IVR interventions to support adherence to medication prescribed for different long-term health conditions. Considering the potential of the IVR to deliver effective interventions, and the evidence on its acceptability to facilitate behaviour change messages, ${ }^{8}$ interventions to promote changes to a single, or multiple, behaviours should be developed and evaluated. Moreover, future studies could usefully test the feasibility, effectiveness and cost-effectiveness of IVR-based interventions for different health behaviours, and in different settings, using rigorous designs and objective outcome measures.

Contributors SS conceived the idea of this review. All authors were involved in developing the methodology of the review. All authors were involved in the data extraction, analysis and interpretation of data, drafting the review and have approved the final version for submission. All authors are accountable for all aspects of the work in ensuring that questions related to the accuracy or integrity of any part of the work are appropriately investigated and resolved.

Funding This study was part of the first author's dissertation in public health at University of Cambridge, funded by the Medical Research Council, UK.

Competing interests None declared.

Patient consent Not required.

Provenance and peer review Not commissioned; externally peer reviewed. 
Data sharing statement Primary data extracted for the meta-analysis and metaregression are available by request to $\mathrm{AK}$ or SS.

Open Access This is an Open Access article distributed in accordance with the Creative Commons Attribution Non Commercial (CC BY-NC 4.0) license, which permits others to distribute, remix, adapt, build upon this work non-commercially, and license their derivative works on different terms, provided the original work is properly cited and the use is non-commercial. See: http://creativecommons.org/ licenses/by-nc/4.0/

(c) Article author(s) (or their employer(s) unless otherwise stated in the text of the article) 2018. All rights reserved. No commercial use is permitted unless otherwise expressly granted

\section{REFERENCES}

1. Danaei G, Ding EL, Mozaffarian D, et al. The preventable causes of death in the United States: comparative risk assessment of dietary, lifestyle, and metabolic risk factors. PLoS Med 2009;6:e1000058.

2. Ford $\mathrm{ES}$, Bergmann MM, Boeing $\mathrm{H}$, et al. Healthy lifestyle behaviors and all-cause mortality among adults in the United States. Prev Med 2012:55:23-7.

3. WHO. Global health risks: mortality and burden of disease attributable to selected major risks: World Health Organisation, 2009. http://www.who.int/healthinfo/global_burden_disease/ GlobalHealthRisks_report_full.pdf

4. NICE. Behaviour change: general approaches; Public health guideline [PH6], 2017.

5. Piette JD, McPhee SJ, Weinberger M, et al. Use of automated telephone disease management calls in an ethnically diverse sample of low-income patients with diabetes. Diabetes Care 1999;22:1302-9.

6. Helzer JE, Rose GL, Badger GJ, et al. Using interactive voice response to enhance brief alcohol intervention in primary care settings. J Stud Alcohol Drugs 2008:69:251-8.

7. Boyce SJ. Natural spoken dialogue systems for telephony applications. Commun ACM 2000;43:29-34.

8. Kassavou A, Sutton S. Reasons for non-adherence to cardiometabolic medications, and acceptability of an interactive voice response intervention in patients with hypertension and type 2 diabetes in primary care: a qualitative study. BMJ Open 2017;7:e015597.

9. Suhm B, Bers J, McCathy D. A comparative study of speech in the call center: natural language call routing vs touch-tone menus. Conference on human factors in computing systems: changing our world, changing ourse/ves. Minneapolis, Minnesota, US.

10. Corkrey R, Parkinson L. Interactive voice response: review of studies 1989-2000. Behav Res Methods Instrum Comput 2002;34:342-53.

11. Piette JD, List J, Rana GK, et al. Mobile health devices as tools for worldwide cardiovascular risk reduction and disease management. Circulation 2015;132:2012-27.

12. Smith DH, O'Keeffe-Rosetti M, Owen-Smith AA, et al. Improving adherence to cardiovascular therapies: an economic evaluation of a randomized pragmatic trial. Value Health 2016;19:176-84

13. Piette JD. Interactive voice response systems in the diagnosis and management of chronic disease. Am J Manag Care 2000;6:817-27.

14. Kassavou A, Sutton S. Automated telecommunication interventions to promote adherence to cardio-metabolic medications: metaanalysis of effectiveness and meta-regression of behaviour change techniques. Health Psychol Rev 2017;12:1-18.

15. Posadzki P, Mastellos N, Ryan R, et al. Automated telephone communication systems for preventive healthcare and management of long-term conditions. Cochrane Database Syst Rev 2016;12:CD009921.

16. Michie S, Richardson M, Johnston M, et al. The behavior change technique taxonomy ( $v 1)$ of 93 hierarchically clustered techniques: building an international consensus for the reporting of behavior change interventions. Ann Behav Med 2013;46:81-95.

17. Michie S, Jochelson K, Markham WA, et al. Low-income groups and behaviour change interventions: a review of intervention content, effectiveness and theoretical frameworks. J Epidemiol Community Health 2009;63:610-22.

18. Reid RD, Pipe AL, Quinlan B, et al. Interactive voice response telephony to promote smoking cessation in patients with heart disease: a pilot study. Patient Educ Couns 2007;66:319-26.

19. Rigotti NA, Japuntich S, Regan S, et al. Promoting smoking cessation after hospital discharge: the helping hand randomized controlled comparative effectiveness trial. J Gen Intern Med 2013:28:S160
20. Alcohol Policy Team UK Department of Health. How to keep health risks from drinking alcohol to a low level. London: Goverment response to the public consultation, 2016.

21. NHS Choice. Eight tips for healthy eating. 2017 http://www.nhs.uk Livewell/Goodfood/Pages/eight-tips-healthy-eating.aspx

22. Department of Health. 2011. Start active, stay active. A report on physical activity for health from the four home countries' Chief Medical Officer. London.

23. Sackett D, Haynes B. Compliance with therapeutic regimens. Baltimore \& London: The John Hopkins University, 1976.

24. Higgins JP GS. Cochrane handbook for systematic reviews of interventions: Cochrane Collaboration, 2011.

25. Andersson C. Comparison of WEB and Interactive Voice Response (IVR) methods for delivering brief alcohol interventions to hazardousdrinking university students: a randomized controlled trial. Eur Addict Res 2015;21:240-52.

26. Sutton S, Kinmonth AL, Hardeman W, et al. Does electronic monitoring influence adherence to medication? Randomized controlled trial of measurement reactivity. Ann Behav Med 2014;48:293-9.

27. French DP, Sutton S. Reactivity of measurement in health psychology: how much of a problem is it? What can be done about it? Br J Health Psychol 2010;15:453-68.

28. Higgins JP, Thompson SG, Deeks JJ, et al. Measuring inconsistency in meta-analyses. BMJ 2003;327:557-60.

29. Egger M, Davey Smith G, Schneider M, et al. Bias in meta-analysis detected by a simple, graphical test. BMJ 1997;315:629-34.

30. Borenstein M, Hedges L, Higgins J, et al. Comprehensive metaanalysis: Proj funded by Natl Institutes Heal, 2017.

31. Cizmic AD, Heilmann RM, Milchak JL, et al. Impact of interactive voice response technology on primary adherence to bisphosphonate therapy: a randomized controlled trial. Osteoporos Int 2015;26:2131-6.

32. Derose SF, Green K, Marrett E, et al. Automated outreach to increase primary adherence to cholesterol-lowering medications. JAMA Intern Med 2013;173:38.

33. Vollmer WM, Owen-Smith AA, Tom JO, et al. Improving adherence to cardiovascular disease medications with information technology. Am J Manag Care 2014;20:SP502.

34. Shet A, De Costa A, Kumarasamy N, et al. Effect of mobile telephone reminders on treatment outcome in HIV: evidence from a randomised controlled trial in India. BMJ 2014;349:95978

35. Sherrard H, Duchesne L, Wells G, et al. Using interactive voice response to improve disease management and compliance with acute coronary syndrome best practice guidelines: A randomized controlled trial. Can J Cardiovasc Nurs 2015;25:10-15

36. Stacy JN, Schwartz SM, Ershoff D, et al. Incorporating tailored interactive patient solutions using interactive voice response technology to improve statin adherence: results of a randomized clinical trial in a managed care setting. Popul Health Manag 2009;12:241-54

37. Vollmer WM, Feldstein A, Smith $\mathrm{DH}$, et al. Use of health information technology to improve medication adherence. Am J Manag Care 2011:17:SP79-87.

38. Sherrard H, Struthers C, Kearns SA, et al. Using technology to create a medication safety net for cardiac surgery patients: a nurse-led randomized control trial. Can J Cardiovasc Nurs 2009;19:9-15.

39. Rose GL, Skelly JM, Badger GJ, et al. Efficacy of automated telephone continuing care following outpatient therapy for alcohol dependence. Addict Behav 2015;41:223-31.

40. King AC, Friedman R, Marcus B, et al. Ongoing physical activity advice by humans versus computers: the Community Health Advice by Telephone (CHAT) trial. Health Psychol 2007;26:718-27.

41. Estabrooks PA, Smith-Ray RL. Piloting a behavioral intervention delivered through interactive voice response telephone messages to promote weight loss in a pre-diabetic population. Patient Educ Couns 2008:72:34-41.

42. Migneault JP, Dedier JJ, Wright JA, et al. A culturally adapted telecommunication system to improve physical activity, diet quality, and medication adherence among hypertensive African-Americans: a randomized controlled trial. Ann Behav Med 2012;43:62-73.

43. Rose GL, Badger GJ, Skelly JM, et al. A randomized controlled tria of brief intervention by interactive voice response. Alcohol Alcohol 2017:52:335-43.

44. Bender BG, Apter A, Bogen DK, et al. Test of an interactive voice response intervention to improve adherence to controller medications in adults with asthma. J Am Board Fam Med 2010;23:159-65.

45. Friedman $\mathrm{RH}$, Stollerman J, Rozenblyum L, et al. A telecommunications system to manage patients with chronic disease. Stud Health Technol Inform 1998:52 Pt 2:1330-4. 
46. Jarvis KL, Friedman RH, Heeren T, et al. Older women and physical activity: using the telephone to walk. Womens Health Issues 1997;7:24-9.

47. Khanna R, Stoddard PJ, Gonzales EN, et al. An automated telephone nutrition support system for Spanish-speaking patients with diabetes. J Diabetes Sci Technol 2014;8:1115-20.

48. Mollon B, Holbrook AM, Keshavjee K, et al. Automated telephone reminder messages can assist electronic diabetes care. J Telemed Telecare 2008;14:32-6.

49. Pai N, Supe P, Kore S, et al. Using automated voice calls to improve adherence to iron supplements during pregnancy. Proc Sixth Int Conf Inf Commun Technol Dev Full Pap - ICTD' 13 2013;1:153-63.

50. Peng W-derB, Schoech D. Evaluation of a web-phone intervention system in changing smoking behavior-a randomized controlled trial. J Technol Hum Serv 2013;31:248-68.
51. Hempel S, Miles JN, Booth MJ, et al. Risk of bias: a simulation study of power to detect study-level moderator effects in meta-analysis. Syst Rev 2013;2:107.

52. DiMatteo MR. Social support and patient adherence to medical treatment: a meta-analysis. Health Psychol 2004;23:207-18.

53. Michie S, Abraham C, Whittington $\mathrm{C}$, et al. Effective techniques in healthy eating and physical activity interventions: a meta-regression. Health Psychol 2009;28:690-701.

54. Black N, Mullan B, Sharpe L. Computer-delivered interventions for reducing alcohol consumption: meta-analysis and meta-regression using behaviour change techniques and theory. Health Psychol Rev 2016;10:341-57.

55. de Bruin M, Viechtbauer W, Hospers HJ, et al. Standard care quality determines treatment outcomes in control groups of HAARTadherence intervention studies: implications for the interpretation and comparison of intervention effects. Health Psychol 2009;28:668-74. 